\title{
Stability of Sampled-Data Control for Lurie Systems with Slope-Restricted Nonlinearities *
}

\author{
Mathias Giordani Titton* João Manoel Gomes da Silva Jr.* \\ Giórgio Valmórbida** \\ * Programa de Pós-Graduação em Engenharia Elétrica (PPGEE), \\ Universidade Federal do Rio Grande do Sul (UFRGS). \\ (e-mails: mathias.titton@ufrgs.br; jmgomes@ufrgs.br) \\ ** Laboratoire des Signaux et Systèmes, CentraleSupélec, \\ CNRS, Université Paris-Saclay. \\ (e-mail: giorgio.valmorbida@l2s.centralesupelec.fr)
}

\begin{abstract}
This paper deals with the stability analysis of aperiodic sampled-data Lurie systems, where the nonlinearity is assumed to be both sector and slope restricted. The proposed method is based on the use of a new class of looped-functionals whose derivative is negative along the trajectories of the continuous-time system. In addition, it contains a generalized Lurie-type function that is quadratic on both the states and the nonlinearity and has a Lurie-Postnikov integral term, which provides some advantages in comparison to simpler candidate functions. On this basis, stability conditions in the form of linear matrix inequalities (LMIs) are formulated. It is shown that the proposed conditions guarantee that the Lurie function is strictly decreasing at the sampling instants, which also implies that the continuous-time trajectories converge asymptotically to the origin. We then formulate some optimization problems for computing the maximal intersampling interval or the maximal sector bounds for which the stability of the sampled-data closed-loop system is guaranteed. A numerical example to illustrate the results is provided.

Resumo: Este artigo trata da análise de estabilidade de sistemas do tipo Lurie com controle amostrado aperiodicamente, onde assume-se que a não linearidade é limitada em setor e em derivada. O método proposto é baseado no uso de uma nova classe de funcionais em loop cuja derivada é negativa ao longo das trajetórias do sistema em tempo contínuo. Além disso, contém uma função generalizada do tipo Lurie que é quadrática tanto nos estados quanto na não linearidade e possui um termo integral de Lurie-Postnikov, oferecendo algumas vantagens em comparação com funções candidatas mais simples. A partir destes elementos, são formuladas condições de estabilidade na forma de inequações lineares matriciais (LMIs). É demonstrado que as condições propostas garantem que a função Lurie seja estritamente decrescente nos instantes de amostragem, o que também implica que as trajetórias em tempo contínuo convergem assintoticamente para a origem. São então formulados alguns problemas de otimização para obter o intervalo máximo entre amostragens ou os limites máximos do setor para os quais a estabilidade do sistema em malha-fechada com controle amostrado é garantida. Os resultados são ilustrados em um exemplo numérico.
\end{abstract}

Keywords: Sampled-data control; Lurie systems; stability analysis; sector bounded nonlinearities; looped-functional approach.

Palavras-chaves: Controle amostrado; sistemas Lurie; análise de estabilidade; não-linearidades limitadas em setor; abordagem looped-functional.

\section{INTRODUCTION}

The study of the stability and stabilization of systems evolving in continuous-time whereas the controller deliver

\footnotetext{
* This study was financed in part by the Coordenação de Aperfeiçoamento de Pessoal de Nível Superior - Brazil (CAPES) - Finance Code 001 and by CNPq (Grants PQ 307449/2019-0 e Univ 422992/20160 ), Brazil. Giorgio Valmorbida is supported by the ANR via grant HANDY, No ANR-18-CE40-0010.
}

inputs at discrete-time instants, i.e., sampled-data control, has been the subject of many works in the literature.

In (Åström and Wittenmark, 2013) linear sampled-data control systems updated at constant sampling periods were extensively studied. However, with the recently growth of networked control applications, asynchronous sampling has been receiving a lot of attention in the literature (Hetel et al., 2017). On the other hand, the fact that for nonlinear systems the exact discretization does not hold, makes the 
stability analysis difficult even in the periodic sampling case. To conclude about the stability of the sampled-data control of nonlinear systems, its hybrid behavior has to be taken into account. Then, it is clear the motivation for developing stability conditions for sampled-data control systems which take into account the variations on the intersampling time as well as nonlinear dynamics.

Different techniques have been proposed in the literature to model sampled-data systems, like the lifting approach (Bamieh et al., 1991), where the problem is transformed into an equivalent finite-dimensional discrete-time problem while keeping the intersampling information of the system; the impulsive modeling, in which a time-varying Lyapunov function is used (Hu et al., 2002), (Naghshtabrizi et al., 2008); the modeling with a continuous-time system with time-varying delay on the plant control input (Fridman et al., 2004), (Fridman, 2010); and the looped-functional approach (Seuret, 2012), (Seuret and Gomes da Silva Jr., 2012) that focus on the behavior in the intersampling interval, ensuring that a positive definite function is strictly decreasing at the sampling instants. For a general overview of these approaches and other ones, the reader can refer to (Hetel et al., 2017).

Much research has been dedicated to Lurie-type nonlinear systems, which consists of a linear time-invariant (LTI) plant connected with a nonlinearity that satisfies some sector restrictions. Considering this class of nonlinear systems, we can mention, for example, works focusing on stabilization (Castelan et al., 2008), Event-Triggered Control (ETC)(Zhang et al., 2019) and synchronization of chaotic systems (Park et al., 2018). Lurie systems with network delays are dealt with in (Hao and Zhao, 2010), (Zeng et al., 2011). Sampled-data systems, which are the focus of this work, were addressed in (Seifullaev and Fradkov, 2013), which uses a Lyapunov-Krasovskii functional and state feedback control law, with extension for robust control in (Seifullaev and Fradkov, 2016). In (Seifullaev and Fradkov, 2015), the authors use an approach from the standpoint of systems passification, with control by linear output feedback.

In these contexts, different classes of Lyapunov Functions (LFs), e.g. quadratic ones (associated to the Circle criterion) (Khalil, 2002), Lurie-Postnikov LFs (associated to the Popov criterion) (Yakubovich, 1965), (Suykens et al., 1998), (Park, 2002) and composite LFs (Hu et al., 2004) have been considered. To deal with sampled-data control systems, Lyapunov-Krasovskii functionals, as proposed in (Fridman, 2010), are in general used. These functionals are required to be positive definite and does not depend on the nonlinearity.

This paper focus on the stability analysis of the sampleddata closed-loop Lurie system. Our approach combines a generalized Lurie function (as Valmorbida et al. (2018)) that does not require the positivity-definiteness of the quadratic part nor the positivity of the Lurie-Postnikov coefficients, with a particular looped-functional (similar to the one used in Seuret and Gomes da Silva Jr. (2012) for input saturated systems).

From these elements, we propose LMI conditions to assess the global asymptotic stability of the origin of the system. In particular, these conditions ensure that a generalized
Lurie function is strictly decreasing at sampling instants. Hence, from the Lurie-type system structure we show that this fact implies the asymptotic convergence of the continuous-time trajectories of the plant to the origin. The LMI conditions can therefore be incorporated as constraints in optimization problems that allow us to compute bounds for the maximal intersampling interval or the maximal sector bounds for which the stability of the Lurie-type system under aperiodic sampled-data control can be ensured.

This paper is organized as follows. The next section describes the problem formulation. Section 3 presents preliminary lemmas for the sector conditions and generalized Lurie-type functions, as well as the functional approach, which will be used to develop the LMI conditions. In Section 4 we derive a theorem to evaluate the asymptotic stability of the closed-loop system under aperiodic sampled-data control. Section 5 features some optimization problems and Section 6 gives a numerical example to highlight the potentialities of the method. Section 7 summarizes at the main points covered in the article.

Notation: The set of diagonal matrices of dimension $n$ is denoted $\mathbb{D}^{n}$ and $\mathbb{D}_{\succ 0}^{n}$ means that the matrix is also positive semi-definite. To express diagonal matrices in a compact form, we write ' $\operatorname{diag}\left(e_{1}, \ldots, e_{n}\right)$ ', where $e_{i}, i=1, \ldots n$ are, respectively, the elements in position $(i, i)$. The set of symmetric matrices of dimension $n$ is denoted $\mathbb{S}^{n}$ and $\mathbb{S}_{\succ 0}^{n}$ means that the matrix is also positive semi-definite. $\bar{A}^{T}$ denotes the transpose of matrix $A . \operatorname{He}(A):=A+A^{T}$, $I_{m}$ and $0_{m}$ are, respectively, the identity and zero square matrices of order $m$. For a given positive scalar, $\mathcal{T}_{2}$, define $\mathbb{K}$ as the set of continuous functions from an interval $[0, Z]$ to $\mathbb{R}^{n}$, where $Z$ is a positive scalar less than $\mathcal{T}_{2}$. The notations $|\cdot|$ and $\|\cdot\|$ represents the absolute value of a scalar and the Euclidean norm, respectively. The $i$ th element of a vector $v$ is denoted by $v_{i} . P \succ 0(P \succeq 0)$ means that $P$ is positive (semi-)definite and $P \prec 0(P \preceq 0)$ means that $P$ is negative (semi-)definite. We denote the time-derivative of a time function, $x$ by $\dot{x}$ and we use $\partial$ to denote the sub-differential operator. The symmetric terms in a symmetric matrix are denoted by $*$. We drop the arguments of some functions when it is clear from the context.

\section{PROBLEM STATEMENT}

Consider the continuous-time plant described by the following Lurie system:

$$
\left\{\begin{array}{l}
\dot{x}(t)=A x(t)+B_{u} u(t)+B_{\phi} \phi(y(t)) \\
y(t)=C x(t)
\end{array},\right.
$$

where $x \in \mathbb{R}^{n}$ represents the states of the plant, $u \in \mathbb{R}^{q}$ represents its input and $y \in \mathbb{R}^{m}$ is the output vector. Matrices $A \in \mathbb{R}^{n \times n}, B_{u} \in \mathbb{R}^{n \times q}, B_{\phi} \in \mathbb{R}^{n \times m}, C \in \mathbb{R}^{m \times n}$, are known and supposed to be constant.

The nonlinearity $\phi: \mathbb{R}^{m} \rightarrow \mathbb{R}^{m}$, is assumed to be time-invariant, memoryless, Lipschitz on $\mathbb{R}^{m}$, decentralized, sector bounded and slope restricted, i.e., $\phi(y)=$ $\left[\phi_{1}\left(y_{1}\right) \ldots \phi_{m}\left(y_{m}\right)\right]^{T}$, with $\phi_{i}\left(y_{i}\right)$ satisfying, $\forall i=1, \ldots, m$

$$
\phi_{i}(0)=0
$$




$$
\begin{gathered}
\frac{\phi_{i}\left(y_{i}\right)}{y_{i}} \in\left[\underline{\delta}_{i}, \bar{\delta}_{i}\right] \quad \forall y \in \mathbb{R}^{m} \\
\partial \phi_{i}\left(y_{i}\right) \in\left[\underline{\gamma}_{i}, \bar{\gamma}_{i}\right], \quad \forall y \in \mathbb{R}^{m},
\end{gathered}
$$

where $\underline{\delta}_{i} \in \mathbb{R}, \bar{\delta}_{i} \in \mathbb{R}, \underline{\delta}_{i} \leq \bar{\delta}_{i}$ are, respectively, the lower and upper sector bounds for the $i$ th nonlinearity and $\underline{\gamma}_{i} \in \mathbb{R}, \bar{\gamma}_{i} \in \mathbb{R}, \underline{\gamma}_{i} \leq \bar{\gamma}_{i}$ are, respectively, the lower and upper slope bounds for the $i$ th nonlinearity. Let us also introduce the matrices $\underline{\Delta}:=\operatorname{diag}\left(\underline{\delta}_{1}, \ldots, \underline{\delta}_{m}\right)$, $\bar{\Delta}:=\operatorname{diag}\left(\bar{\delta}_{1}, \ldots, \bar{\delta}_{m}\right), \quad \underline{\Gamma}:=\operatorname{diag}\left(\underline{\gamma}_{1}, \ldots, \underline{\gamma}_{m}\right), \bar{\Gamma}:=$ $\operatorname{diag}\left(\bar{\gamma}_{1}, \ldots, \bar{\gamma}_{m}\right)$ to express the sector and slope conditions in matrix format. The Lipschitz assumption on $\phi$ implies that $\partial \phi_{i}\left(y_{i}\right)=\frac{d \phi_{i}}{d y_{i}}$ almost everywhere, relaxing the requirement for the nonlinearity to be continuously differentiable.

We suppose that the control signal $u$ comes from a digital controller and it is updated at sampling instants $t=$ $t_{k}$, remaining constant between two successive sampling instants through a zero-order hold (ZOH). Thus, $\left\{t_{k}\right\}_{k \in \mathbb{N}}$ is an increasing sequence of positive scalars such that $\bigcup_{k \in \mathbb{N}}\left[t_{k}, t_{k+1}\right)=[0,+\infty)$. Since we are considering the generic case of aperiodic sampling, assume that there exist two positive scalars $\mathcal{T}_{1} \leq \mathcal{T}_{2}$ such that the difference between two successive sampling instants $T_{k}=t_{k+1}-t_{k}$ satisfies

$$
0<\mathcal{T}_{1} \leq T_{k} \leq \mathcal{T}_{2}, \quad \forall k \in \mathbb{N} .
$$

Note that in periodic sampling we assume in particular that $T_{k}$ must have the same value $\forall k \in \mathbb{N}$, i.e., $T_{k}=\mathcal{T}_{1}=$ $\mathcal{T}_{2}, \forall k$.

In particular, we consider the following generic sampleddata control law:

$$
\begin{aligned}
u(t) & =K_{x} x\left(t_{k}\right)+K_{\phi} \phi\left(y\left(t_{k}\right)\right) \\
& =K_{x} x\left(t_{k}\right)+K_{\phi} \phi\left(C x\left(t_{k}\right)\right), \quad \forall t \in\left[t_{k}, t_{k+1}\right),
\end{aligned}
$$

where $K_{x} \in \mathbb{R}^{q \times n}, K_{\phi} \in \mathbb{R}^{q \times m}$. This control law allows to use information about the nonlinearities (Castelan et al., 2008). If nonlinearities cannot be measured or if the control law is simply a state-feedback, just consider $K_{\phi}=0$.

In this paper, we are interested in formulating conditions that allow us to evaluate the global asymptotic stability of the system (1) with nonlinearities satisfying sector and slope restrictions (2), subject to aperiodic sampling (3) and control law given by (4).

\section{PRELIMINARIES}

In this section, we present the inequalities verified by the sector and slope bounded nonlinearity. These inequalities will play a very important role to obtain conditions to assess the stability of the closed-loop system (1)-(4).

\subsection{Sector Conditions}

Define $S_{\Delta}: \mathbb{R}^{m \times m} \times \mathbb{R}^{m} \times \mathbb{R}^{m} \rightarrow \mathbb{R}, S_{\Gamma}: \mathbb{R}^{m \times m} \times \mathbb{R}^{m} \times$ $\mathbb{R}^{m} \rightarrow \mathbb{R}$ as

$$
\begin{aligned}
S_{\Delta}(U, \phi, \kappa) & :=(\phi-\underline{\Delta} \kappa)^{T} U(\bar{\Delta} \kappa-\phi) \\
S_{\Gamma}(U, \phi, \kappa) & :=(\phi-\underline{\Gamma} \kappa)^{T} U(\bar{\Gamma} \kappa-\phi) .
\end{aligned}
$$

Lemma 1. (Khalil, 2002). If $U_{1} \in \mathbb{D}_{\succeq 0}^{m}$ and $\phi: \mathbb{R}^{m} \rightarrow \mathbb{R}^{m}$ satisfies (2), then

$$
S_{\Delta}\left(U_{1}, \phi(\kappa), \kappa\right) \geq 0
$$

for all $\kappa \in \mathbb{R}^{m}$.

Lemma 2. (Valmorbida et al., 2018). If $U_{2} \in \mathbb{D}_{\succeq 0}^{m}$ and $\phi: \mathbb{R}^{m} \rightarrow \mathbb{R}^{m}$ satisfies (2), then

$$
S_{\Gamma}\left(U_{2}, \dot{\phi}(\kappa), \dot{\kappa}\right) \geq 0
$$

almost everywhere for $\kappa \in \mathbb{R}^{m}$.

\subsection{Looped-Functional Approach}

Define, as in (Seuret, 2012), $x_{k}(\tau):=x\left(t_{k}+\tau\right), y_{k}(\tau):=$ $y\left(t_{k}+\tau\right)$ and $\phi_{k}(\tau):=\phi\left(y_{k}(\tau)\right)$. Hence, the behavior of the closed-loop system in the interval $\left[t_{k}, t_{k+1}\right)$ can be described by:

$\left\{\begin{aligned} & \dot{x}_{k}(\tau)=A x_{k}(\tau)+B_{\phi} \phi_{k}(\tau)+B_{u}\left(K_{x} x_{k}(0)+K_{\phi} \phi_{k}(0)\right) \\ & y_{k}(\tau)=C x_{k}(\tau) \forall \tau \in\left[0, T_{k}\right),\end{aligned}\right.$

where $\dot{x}_{k}(\tau)=\frac{d}{d \tau} x_{k}(\tau)$. Using representation (7), and inspired by the results in Seuret and Gomes da Silva Jr. (2012) considering linear sampled-data control systems subject to actuator saturation, the following theorem regarding the looped-functional approach to assess the closed-loop stability of a Lurie-type system can be stated.

Theorem 1. Let $V: \mathbb{R}^{n} \rightarrow \mathbb{R}^{+}$be a differentiable function such that

$$
\mu_{1}\|x\|^{p} \leq V(x) \leq \mu_{2}\|x\|^{p}, \forall x \in \mathbb{R}^{n},
$$

with $\mu_{1}, \mu_{2}>0, p>0$, and let $\mathcal{W}_{0}:\left[0, \mathcal{T}_{2}\right] \times \mathbb{K} \times$ $\left[\mathcal{T}_{1}, \mathcal{T}_{2}\right] \rightarrow \mathbb{R}$ be a continuous and differentiable functional, called a looped-functional, that satisfies for all $z \in \mathbb{K}$ and for all $T_{k} \in\left[\mathcal{T}_{1}, \mathcal{T}_{2}\right]$

$$
\mathcal{W}_{0}\left(T_{k}, z, T_{k}\right)=\mathcal{W}_{0}\left(0, z, T_{k}\right)
$$

If for all $k \in \mathbb{N}, T_{k} \in\left[\mathcal{T}_{1}, \mathcal{T}_{2}\right]$ and $\tau \in\left[0, T_{k}\right]$ one has

$$
\dot{W}\left(\tau, x_{k}, T_{k}\right)=\frac{d}{d \tau}\left[V\left(x_{k}(\tau)\right)+\mathcal{W}_{0}\left(\tau, x_{k}, T_{k}\right)\right]<0,
$$

then considering the solutions of the system (1) with the sampled-data control law (4) it follows that

(i) $\Delta V(k)=V\left(x_{k+1}(0)\right)-V\left(x_{k}(0)\right)=V\left(x\left(t_{k+1}\right)\right)-$ $\left.V\left(x\left(t_{k}\right)\right)\right)<0, \forall k \in \mathbb{N}$

(ii) $x(t) \rightarrow 0$ as $t \rightarrow \infty, \forall x(0) \in \mathbb{R}^{n}$, i.e. the origin is globally asymptotically stable (GAS).

Proof. First note that, by continuity of the system trajectories $x_{k}\left(T_{k}\right)=x_{k+1}(0)$. Integrating now (10) in the interval $\left[0, T_{k}\right]$ and using (9), we conclude that this inequality implies that $\Delta V(k)=V\left(x_{k+1}(0)\right)-V\left(x_{k}(0)\right)=$ $V\left(x\left(t_{k+1}\right)\right)-V\left(x\left(t_{k}\right)\right)<0, \forall k \in \mathbb{N}$. Thus, we can conclude that $x_{k}(0)=x\left(t_{k}\right) \rightarrow 0$ as $k \rightarrow \infty$.

To conclude the proof, we need to show that the continuous-time trajectories also converge to the origin, i.e., $x(t) \rightarrow 0$ as $t \rightarrow \infty$ and that $x(t)$ is uniformly bounded for all $t \in\left[t_{k}, t_{k+1}\right), \forall k$.

For this, note that as $\frac{\phi_{i}\left(y_{i}\right)}{y_{i}} \in\left[\underline{\delta}_{i}, \bar{\delta}_{i}\right]$, it follows that, $\forall t$, there exists a scalar $\alpha(t), 0 \leq \alpha(t)<1$ such that

$$
\begin{aligned}
\phi_{i}\left(y_{i}(t)\right) & =\left[\alpha(t) \underline{\delta}_{i}+(1-\alpha(t)) \bar{\delta}_{i}\right] y_{i}(t) \\
& =v_{i}(t) y_{i}(t), \quad \text { with } v_{i}(t) \in\left[\underline{\delta}_{i}, \bar{\delta}_{i}\right] .
\end{aligned}
$$


Thus, we can write $\phi(y(t))=\Upsilon(v(t)) y(t)$, with $\Upsilon(v(t))=$ $\operatorname{diag}\left(v_{1}(t), v_{2}(t), \ldots, v_{m}(t)\right), v(t) \in \mho_{v}=\left\{v \in \mathbb{R}^{m} \mid \underline{\delta}_{i}<\right.$ $\left.v_{i}<\bar{\delta}_{i}, i=1, \ldots, m\right\}$. Hence, the closed-loop system can be represented by the following linear time-varying system:

$$
\begin{aligned}
\dot{x}(t)=A x(t) & +B_{u}\left(K_{x} x\left(t_{k}\right)+K_{\phi} \phi\left(y\left(t_{k}\right)\right)\right. \\
+ & B_{\phi} \Upsilon(v(t)) C x(t), \forall t \in\left[t_{k}, t_{k+1}\right) .
\end{aligned}
$$

Considering the lifted-variables one has

$$
\begin{aligned}
\dot{x}_{k}(\tau)=\left(A+B_{\phi} \Upsilon\right. & \left.\left(v_{k}(\tau)\right) C\right) x_{k}(\tau) \\
& +B_{u}\left(K_{x} x_{k}(0)+K_{\phi} \phi_{k}(0)\right) .
\end{aligned}
$$

For each admissible $v(t) \in \mho_{v}$, we can define a transition matrix $\Psi_{v}\left(t, t_{0}\right)$ for system (12). Defining $v_{k}(\tau):=v\left(t_{k}+\right.$ $\tau), \Psi_{v_{k}}(\tau):=\Psi_{v}\left(t_{k}+\tau, t_{k}\right)$ as the restriction of $\Psi_{v}\left(t, t_{0}\right)$ to the interval $\left[t_{k}, t_{k+1}\right)$, and the state transition matrix $\Psi_{v_{k}}(\tau, s):=\Psi_{v}\left(t_{k}+\tau, t_{k}+s\right)$, it follows that

$x_{k}(\tau)=\Psi_{v_{k}}(\tau, 0) x_{k}(0)+\int_{0}^{\tau} \Psi_{v_{k}}(\tau, s) B_{u}\left[K_{x} K_{\phi}\right]\left[\begin{array}{l}x_{k}(0) \\ \phi_{k}(0)\end{array}\right] d s$.

Thus, we have that

$$
\begin{aligned}
\left\|x_{k}(\tau)\right\| \leq & \left\|\Psi_{v_{k}}(\tau, 0)\right\|\left\|x_{k}(0)\right\|+\left\|\int_{0}^{\tau} \Psi_{v_{k}}(\tau, s) d s\right\| \times \\
& \left\|B_{u}\left[K_{x} K_{\phi}\right]\left[\begin{array}{c}
x_{k}(0) \\
\phi_{k}(0)
\end{array}\right]\right\| \\
\leq & \left(\left\|\Psi_{v_{k}}(\tau, 0)\right\|+\left\|\int_{0}^{\tau} \Psi_{v_{k}}(\tau, s) d s\right\|\left(\left\|B_{u} K_{x}\right\|\right.\right. \\
& \left.\left.+\left\|B_{u} K_{\phi}\right\|\|\bar{\Delta} C\|\right)\right)\left\|x_{k}(0)\right\| \\
\leq & \left(\left\|\Psi_{v_{k}}(\tau, 0)\right\|+\int_{0}^{\mathcal{T}_{2}}\left\|\Psi_{v_{k}}(\tau, s) d s\right\|\left(\left\|B_{u} K_{x}\right\|\right.\right. \\
& \left.\left.+\left\|B_{u} K_{\phi} \bar{\Delta} C\right\|\right)\right)\left\|x_{k}(0)\right\| .
\end{aligned}
$$

As $v_{k}(\tau) \in \mho_{v}, \forall \tau \in\left[0, \mathcal{T}_{2}\right]$, there exists a scalar $\mu_{\Psi}$, such that

$$
\left\|x_{k}(\tau)\right\| \leq \mu_{\Psi}\left\|x_{k}(0)\right\|
$$

where

$$
\begin{array}{r}
\mu_{\Psi}=\sup _{v_{k}(\tau) \in \mho_{v}}\left(\left\|\Psi_{v_{k}}(\tau, 0)\right\|+\int_{0}^{\mathcal{T}_{2}}\left\|\Psi_{v_{k}}(\tau, s) d s\right\| \times\right. \\
\left.\left(\left\|B_{u} K_{x}\right\|+\left\|B_{u} K_{\phi}\right\|\|\bar{\Delta}\|\|C\|\right)\right) .
\end{array}
$$

Hence, if $x_{k}(0) \rightarrow 0$ as $k \rightarrow \infty$, i.e., $x\left(t_{k}\right) \rightarrow 0$ as $k \rightarrow \infty$, then $x\left(t_{k}+\tau\right)=x_{k}(\tau) \rightarrow 0$ as $k \rightarrow \infty, \forall \tau \in\left[0, T_{k}\right]$, that is $x(t)=x\left(t_{k}+\tau\right) \rightarrow 0$ as $t \rightarrow \infty$.

\subsection{Generalized Lurie Function}

Consider a generalized Lurie function $V: \mathbb{R}^{n} \rightarrow \mathbb{R}$ defined as follows

$$
V(x)=V_{0}(x)+\sum_{i=1}^{m} \lambda_{i} \int_{0}^{y_{i}}\left(\phi_{i}(s)-\underline{\delta}_{i} s\right) d s,
$$

where $y_{i}=(C x)_{i}, i=1, \ldots, m$ and

$$
V_{0}(x)=\left[\begin{array}{c}
x \\
\phi(y)
\end{array}\right]^{T}\left[\begin{array}{ll}
P_{11} & P_{12} \\
P_{12}^{T} & P_{22}
\end{array}\right]\left[\begin{array}{c}
x \\
\phi(y)
\end{array}\right] \text {. }
$$

This function is an extended version of the traditional Lurie function, where the matrix $P$ and the coefficients $\lambda_{i}$ (also known as Lurie-Postnikov terms), $\Lambda:=$ $\operatorname{diag}\left(\lambda_{1}, \ldots, \lambda_{i}\right), i=1, \ldots, m$, are necessarily positivedefinite. Note that this form does not require the positivedefiniteness of $P$, nor the non-negativity of the coefficients $\lambda_{i}$ (Valmorbida et al., 2018). In this case, the positivitydefiniteness of $V$ should be ensured by other means, as stated in the following lemma.

Lemma 3. (Valmorbida et al., 2018). Consider $V$ in (17) where $\phi$ satisfies $(2 \mathrm{a})-(2 \mathrm{~b})$. If there exists a matrix $\tilde{\Lambda} \in \mathbb{D}_{\succeq 0}^{m}$ such that

$$
\begin{aligned}
& \Lambda \succeq-\tilde{\Lambda}, \\
& V_{0}(x)-\frac{1}{2} y^{T}(\bar{\Delta}-\underline{\Delta}) \tilde{\Lambda} y>0, \quad \forall x \in \mathbb{R}^{n},
\end{aligned}
$$

then $V(x)>0, \forall x \in \mathbb{R}^{n}$.

\section{ASYMPTOTIC STABILITY CONDITIONS}

In this section, we derive conditions in the form of linear matrix inequalities (LMIs) to assess the asymptotic stability of the sampled-data closed-loop system (1)-(4). The approach is based on the use of looped-functionals (Seuret and Gomes da Silva Jr., 2012) and a generalized Lurietype function (Valmorbida et al., 2018). With this aim, we apply Theorem 1, considering the function $V(x)$ defined in (17) and the functional

$$
\begin{aligned}
& \mathcal{W}_{0}\left(\tau, x_{k}, T_{k}\right)=\left(T_{k}-\tau\right)\left\{\int_{0}^{\tau} \dot{x}_{k}^{T}(\theta) R_{x} \dot{x}_{k}(\theta) d \theta\right. \\
& \left.+\left(x_{k}(\tau)-x_{k}(0)\right)^{T}\left[F_{x}\left(x_{k}(\tau)-x_{k}(0)\right)+2 G_{x} x_{k}(0)\right]\right\} \\
& +\left(T_{k}-\tau\right)\left\{\tau\left[\begin{array}{l}
x_{k}(0) \\
\phi_{k}(0)
\end{array}\right]^{T} X\left[\begin{array}{l}
x_{k}(0) \\
\phi_{k}(0)
\end{array}\right]+\int_{0}^{\tau} \dot{\phi}_{k}^{T}(\theta) R_{\phi} \dot{\phi}_{k}(\theta) d \theta\right. \\
& \left.+\left(\phi_{k}(\tau)-\phi_{k}(0)\right)^{T}\left[F_{\phi}\left(\phi_{k}(\tau)-\phi_{k}(0)\right)+2 G_{\phi} \phi_{k}(0)\right]\right\}
\end{aligned}
$$

with $F_{x} \in \mathbb{S}^{n}, G_{x} \in \mathbb{R}^{n \times n}, X \in \mathbb{S}^{n+m}, F_{\phi} \in \mathbb{S}^{m}$, $G_{\phi} \in \mathbb{R}^{m \times m}, R_{x} \in \mathbb{S}_{\succ 0}^{n}$ and $R_{\phi} \in \mathbb{S}_{\succ 0}^{m}$.

The following theorem provides LMI conditions to verify the stability of the closed-loop system under aperiodic sampling.

Theorem 2. Assume that there exist a matrix $P \in \mathbb{S}^{n+m}$, matrices $\Lambda \in \mathbb{D}^{m}, \tilde{\Lambda} \in \mathbb{D}_{\succeq 0}^{m}$, matrices $U_{j}, j=0, \ldots, 3 \in$ $\mathbb{D}_{\succeq 0}^{m}$, matrices $F_{x} \in \mathbb{S}^{n}, G_{x} \in \mathbb{R}^{n \times n}, F_{\phi} \in \mathbb{S}^{m}, G_{\phi} \in$ $\mathbb{R}^{m \times m}, R_{x} \in \mathbb{S}_{\succ 0}^{n}, R_{\phi} \in \mathbb{S}_{\succ 0}^{m}, Q_{x} \in \mathbb{R}^{(3 n) \times n}, Q_{\phi} \in$ $\mathbb{R}^{(3 m) \times m}, X \in \mathbb{S}^{n+m}, Y_{1}, Y_{2} \in \mathbb{R}^{n \times n}$ that satisfy, for $i=1,2$ :

$$
\begin{aligned}
\Psi_{1}\left(\mathcal{T}_{i}\right) & =\Pi_{1}+\mathcal{T}_{i} \Pi_{2}+\mathcal{T}_{i} \Pi_{3} \prec 0 \\
\Psi_{2}\left(\mathcal{T}_{i}\right) & =\left[\begin{array}{ccc}
\Pi_{1}-\mathcal{T}_{i} \Pi_{3} & \mathcal{T}_{i} M_{135}^{T} Q_{x} & \mathcal{T}_{i} M_{246}^{T} Q_{\phi} \\
* & -\mathcal{T}_{i} R_{x} & 0 \\
* & * & -\mathcal{T}_{i} R_{\phi}
\end{array}\right] \prec 0
\end{aligned}
$$




$$
\begin{aligned}
& \Lambda \succeq-\tilde{\Lambda} \\
& P-\frac{1}{2}\left[\begin{array}{c}
C^{T} \\
0
\end{array}\right](\bar{\Delta}-\underline{\Delta}) \tilde{\Lambda}\left[\begin{array}{ll}
C & 0
\end{array}\right] \\
& +\operatorname{He}\left\{\frac{1}{2}\left[\begin{array}{c}
(\underline{\Delta} C)^{T} \\
-I_{m}
\end{array}\right] U_{0}\left[\bar{\Delta} C-I_{m}\right]\right\} \succ 0,
\end{aligned}
$$

with

$$
\begin{aligned}
\Pi_{1} & =\operatorname{He}\left\{M_{12}^{T} P M_{34}\right\}-M_{15}^{T} F_{x} M_{15}-\operatorname{He}\left\{M_{15}^{T} G_{x} M_{5}\right\} \\
& -\operatorname{He}\left\{\frac{1}{2}\left(M_{1}^{T}(\underline{\Delta} C)^{T}-M_{2}^{T}\right) \Lambda C M_{3}\right\} \\
& -M_{26}^{T} F_{\phi} M_{26}-\operatorname{He}\left\{M_{26}^{T} G_{\phi} M_{6}\right\} \\
& \left.+\operatorname{He}\left\{M_{2}^{T}-M_{1}^{T}(\underline{\Delta} C)^{T}\right) U_{1}\left(\bar{\Delta} C M_{1}-M_{2}\right)\right\} \\
& \left.+\operatorname{He}\left\{M_{4}^{T}-M_{3}^{T}(\underline{\Gamma} C)^{T}\right) U_{2}\left(\bar{\Gamma} C M_{3}-M_{4}\right)\right\} \\
& \left.+\operatorname{He}\left\{M_{6}^{T}-M_{5}^{T}(\underline{\Delta} C)^{T}\right) U_{3}\left(\bar{\Delta} C M_{5}-M_{6}\right)\right\} \\
& -\operatorname{He}\left\{M_{135}^{T} Q_{x} M_{15}\right\}-\operatorname{He}\left\{M_{246}^{T} Q_{\phi} M_{26}\right\} \\
& +\operatorname{He}\left\{\left(M_{1}^{T} Y_{1}^{T}+M_{3}^{T} Y_{2}^{T}\right) M_{0}\right\} \\
\Pi_{2} & =M_{3}^{T} R_{x} M_{3}+\operatorname{He}\left\{M_{3}^{T}\left(F_{x} M_{15}+G_{x} M_{5}\right)\right\} \\
& +M_{4}^{T} R_{\phi} M_{4}+\operatorname{He}\left\{M_{4}^{T}\left(F_{\phi} M_{26}+G_{\phi} M_{6}\right)\right\} \\
\Pi_{3} & =M_{56}^{T} X M_{56},
\end{aligned}
$$

where $^{1}$

$$
\begin{aligned}
& M_{0}=\left[\begin{array}{llllll}
A & B_{\phi} & -I & 0 & B_{u} K_{x} & B_{u} K_{\phi}
\end{array}\right] \\
& M_{1}=\left[\begin{array}{llllll}
I & 0 & 0 & 0 & 0 & 0
\end{array}\right] \quad M_{2}=\left[\begin{array}{llllll}
0 & I & 0 & 0 & 0 & 0
\end{array}\right] \\
& M_{3}=\left[\begin{array}{llllll}
0 & 0 & I & 0 & 0 & 0
\end{array}\right] \quad M_{4}=\left[\begin{array}{llllll}
0 & 0 & 0 & I & 0 & 0
\end{array}\right] \\
& M_{5}=\left[\begin{array}{llllll}
0 & 0 & 0 & 0 & I & 0
\end{array}\right] \quad M_{6}=\left[\begin{array}{llllll}
0 & 0 & 0 & 0 & 0 & I
\end{array}\right] \\
& M_{15}=M_{1}-M_{5} \quad M_{26}=M_{2}-M_{6} \\
& M_{12}=\left[\begin{array}{ll}
M_{1}^{T} & M_{2}^{T}
\end{array}\right]^{T} \quad M_{34}=\left[\begin{array}{ll}
M_{3}^{T} & M_{4}^{T}
\end{array}\right]^{T} \\
& M_{56}=\left[\begin{array}{ll}
M_{5}^{T} & M_{6}^{T}
\end{array}\right]^{T} \quad M_{135}=\left[\begin{array}{lll}
M_{1}^{T} & M_{3}^{T} & M_{5}^{T}
\end{array}\right]^{T} \\
& M_{246}=\left[\begin{array}{lll}
M_{2}^{T} & M_{4}^{T} & M_{6}^{T}
\end{array}\right]^{T} \text {. }
\end{aligned}
$$

Then the origin of the sampled-data closed-loop system (1)-(4) is GAS.

Proof. Considering the result of Theorem 1, the idea is to prove that (9) and (10) are satisfied, $\forall k \in \mathbb{N}$, considering $V$ and $\mathcal{W}_{0}$ as given in (17) and (21), respectively.

It is straightforward to proof the positivity of $V$ given by the inequalities (24) and (25) using Lemma 1 and Lemma 3. The next step of the proof focuses on the fact that the functional $\mathcal{W}_{0}$ given in (21) satisfies the condition (9). Since $\left(T_{k}-\tau\right)=0$ when $\tau=T_{k}$ and $x_{k}(\tau)-x_{k}(0)=0$ when $\tau=0$, it follows that the functional $\mathcal{W}_{0}$ satisfies $(9)$. Moreover, it is continuous at all sampling instants and differentiable over $\left[0, T_{k}\right)$. The rest of the proof consists in showing that (22)-(23) implies (10). With this aim, the expression of $\dot{W}$ is given as follows:

\footnotetext{
1 The matrices $M_{i}$ are not of the same dimension. The notations 0 and $I$ correspond to the zero and identity matrices of appropriate dimension.
}

$$
\begin{aligned}
& \dot{W}\left(\tau, x_{k}, T_{k}\right)=2\left[\begin{array}{c}
x_{k}(\tau) \\
\phi_{k}(\tau)
\end{array}\right]^{T} P\left[\begin{array}{c}
\dot{x}_{k}(\tau) \\
\dot{\phi}_{k}(\tau)
\end{array}\right] \\
& -\left[\left(x_{k}^{T}(\tau)(\underline{\Delta} C)^{T}-\phi_{k}^{T}(\tau)\right) \Lambda C\right] \dot{x}_{k}(\tau) \\
& -\left(x_{k}(\tau)-x_{k}(0)\right)^{T}\left[F_{x}\left(x_{k}(\tau)-x_{k}(0)\right)+2 G_{x} x_{k}(0)\right] \\
& +\left(T_{k}-\tau\right) \dot{x}_{k}^{T}(\tau)\left[R_{x} \dot{x}_{k}(\tau)+2 F_{x}\left(x_{k}(\tau)-x_{k}(0)\right)\right. \\
& \left.+2 G_{x} x_{k}(0)\right]+\left(T_{k}-2 \tau\right)\left[\begin{array}{l}
x_{k}(0) \\
\phi_{k}(0)
\end{array}\right]^{T} X\left[\begin{array}{l}
x_{k}(0) \\
\phi_{k}(0)
\end{array}\right] \\
& -\left(\phi_{k}(\tau)-\phi_{k}(0)\right)^{T}\left[F_{\phi}\left(\phi_{k}(\tau)-\phi_{k}(0)\right)+2 G_{\phi} \phi_{k}(0)\right] \\
& +\left(T_{k}-\tau\right) \dot{\phi}_{k}^{T}(\tau)\left[R_{\phi} \dot{\phi}_{k}(\tau)+2 F_{\phi}\left(\phi_{k}(\tau)-\phi_{k}(0)\right)\right. \\
& \left.+2 G_{\phi} \phi_{k}(0)\right]-\int_{0}^{\tau} \dot{x}_{k}^{T}(\theta) R_{x} \dot{x}_{k}(\theta) d \theta \\
& -\int_{0}^{\tau} \dot{\phi}_{k}^{T}(\theta) R_{\phi} \dot{\phi}_{k}(\theta) d \theta .
\end{aligned}
$$

Using the sector conditions defined in Lemma 1 and Lemma 2, we have that

$$
\begin{aligned}
& \dot{W}\left(\tau, x_{k}, T_{k}\right)<\dot{W}\left(\tau, x_{k}, T_{k}\right)+2 S_{\Delta}\left(U_{1}, \phi_{k}(\tau), y_{k}(\tau)\right) \\
& +2 S_{\Gamma}\left(U_{2}, \dot{\phi}_{k}(\tau), \dot{y}_{k}(\tau)\right)+2 S_{\Delta}\left(U_{3}, \phi_{k}(0), y_{k}(0)\right),
\end{aligned}
$$

or equivalently

$$
\begin{aligned}
& \dot{W}\left(\tau, x_{k}, T_{k}\right)<\dot{W}\left(\tau, x_{k}, T_{k}\right) \\
& +2\left(\phi_{k}^{T}(\tau)-x_{k}^{T}(\tau)(\underline{\Delta} C)^{T}\right) U_{1}\left(\bar{\Delta} C x_{k}(\tau)-\phi_{k}(\tau)\right) \\
& +2\left(\dot{\phi}_{k}^{T}(\tau)-\dot{x}_{k}^{T}(\tau)(\underline{\Gamma} C)^{T}\right) U_{2}\left(\bar{\Gamma} C \dot{x}_{k}(\tau)-\dot{\phi}_{k}(\tau)\right) \\
& +2\left(\phi_{k}^{T}(0)-x_{k}^{T}(0)(\underline{\Delta} C)^{T}\right) U_{3}\left(\bar{\Delta} C x_{k}(0)-\phi_{k}(0)\right) .
\end{aligned}
$$

Consider the vector $\eta_{k}(\tau)=\left[x_{k}^{T}(\tau) \phi_{k}^{T}(\tau) \dot{x}_{k}^{T}(\tau) \dot{\phi}_{k}^{T}(\tau)\right.$ $\left.x_{k}^{T}(0) \phi_{k}^{T}(0)\right]^{T}$, the vector $\zeta_{k}(\tau)=M_{135} \eta_{k}(\tau)$ and a matrix $Q_{x} \in \mathbb{R}^{(3 n) \times n}$. Since $R_{x}$ is assumed to be positive definite, it follows that $\left(\dot{x}_{k}(\theta)-R_{x}^{-1} Q_{x}^{T} \zeta_{k}(\tau)\right)^{T} R_{x}\left(\dot{x}_{k}(\theta)-\right.$ $\left.R_{x}^{-1} Q_{x}^{T} \zeta_{k}(\tau)\right)>0$. Integrating this expression over $[0, \tau]$, the following inequality is obtained

$$
\begin{gathered}
\int_{0}^{\tau} \dot{x_{k}}(\theta)^{T} R_{x} \dot{x_{k}}(\theta) d \theta-2 \zeta_{k}^{T}(\tau) Q_{x}\left(x_{k}(\tau)-x_{k}(0)\right) \\
+\tau \zeta_{k}^{T}(\tau) Q_{x} R_{x}^{-1} Q_{x}^{T} \zeta_{k}(\tau) \geq 0
\end{gathered}
$$

Consider now a new vector $\psi_{k}(\tau)=M_{246} \eta_{k}(\tau)$ and a matrix $Q_{\phi} \in \mathbb{R}^{(3 m) \times m}$. Following the same reasoning as above, we have that:

$$
\begin{gathered}
\int_{0}^{\tau} \dot{\phi}_{k}(\theta)^{T} R_{\phi} \dot{\phi}_{k}(\theta) d \theta-2 \psi_{k}^{T}(\tau) Q_{\phi}\left(\phi_{k}(\tau)-\phi_{k}(0)\right) \\
+\tau \psi_{k}^{T}(\tau) Q_{\phi} R_{\phi}^{-1} Q_{\phi}^{T} \psi_{k}(\tau) \geq 0 .
\end{gathered}
$$

On the other hand, from (7), there exists a coupling relation between the components of the vector $\eta_{k}(\tau)$. Hence, $\forall Y_{1} \in \mathbb{R}^{n \times n}$ and $\forall Y_{2} \in \mathbb{R}^{n \times n}$ the following equality is satisfied:

$$
\begin{gathered}
2\left(x_{k}^{T}(\tau) Y_{1}^{T}+\dot{x}_{k}^{T}(\tau) Y_{2}^{T}\right)\left(A x_{k}(\tau)+B_{\phi} \phi_{k}(\tau)\right. \\
\left.-\dot{x}_{k}(\tau)+B_{u}\left(K_{x} x_{k}(0)+K_{\phi} \phi_{k}(0)\right)\right)=0 .
\end{gathered}
$$

This null term can be added to (27). Hence, combining (27), (28), (29) and (30), one obtains that

$$
\begin{aligned}
\dot{W} & \leq \eta_{k}^{T}(\tau)\left[\Pi_{1}+\left(T_{k}-\tau\right) \Pi_{2}+\tau\left(M_{135}^{T} Q_{x} R_{x}^{-1} Q_{x}^{T} M_{135}\right.\right. \\
& \left.\left.+M_{246}^{T} Q_{\phi} R_{\phi}^{-1} Q_{\phi}^{T} M_{246}\right)+\left(T_{k}-2 \tau\right) \Pi_{3}\right] \eta_{k}(\tau) .
\end{aligned}
$$

Thus, to prove that $\dot{W}<0$, it suffices to guarantee that 


$$
\begin{aligned}
& \Pi_{1}+\left(T_{k}-\tau\right) \Pi_{2}+\tau\left(M_{135}^{T} Q_{x} R_{x}^{-1} Q_{x}^{T} M_{135}\right. \\
& \left.+M_{246}^{T} Q_{\phi} R_{\phi}^{-1} Q_{\phi}^{T} M_{246}\right)+\left(T_{k}-2 \tau\right) \Pi_{3} \prec 0 .
\end{aligned}
$$

As this matrix inequality is affine with respect to $\tau$, and $\tau \in\left[0, T_{k}\right]$, a necessary and sufficient condition to satisfy it, is given by

$$
\left\{\begin{aligned}
& \Pi_{1}+T_{k}\left(\Pi_{2}+\Pi_{3}\right) \prec 0 \\
& \Pi_{1}-T_{k} \Pi_{3}+ T_{k}\left(M_{135}^{T} Q_{x} R_{x}^{-1} Q_{x}^{T} M_{135}\right. \\
&\left.+M_{246}^{T} Q_{\phi} R_{\phi}^{-1} Q_{\phi}^{T} M_{246}\right) \prec 0 .
\end{aligned}\right.
$$

Finally, as (31) and (32) are affine in $T_{k}$ and $T_{k} \in\left[\mathcal{T}_{1}, \mathcal{T}_{2}\right]$, applying the same reasoning and the Schur complement to $(32)$, we conclude that $\Psi_{1}\left(\mathcal{T}_{i}\right) \prec 0$ and $\Psi_{2}\left(\mathcal{T}_{i}\right) \prec 0$, $i=1,2$, are sufficient to ensure $\dot{W}<0$. Hence, by virtue of Theorem 1 the satisfaction of conditions (22)-(25) ensures the global asymptotic convergence of the trajectories to the origin.

\section{OPTIMIZATION PROBLEMS}

From the conditions stated in Theorem 2, we can formulate three optimization problems as follows.

P1. Given $\mathcal{T}_{1}$, the sector and slope bounds, find the maximal $\mathcal{T}_{2}$ such that global stability of the closedloop system (7) is ensured.

P2. Given a nominal sampling time $\mathcal{T}_{\text {nom }}$, the sector and slope bounds, find the maximum symmetrical boundaries (Jitter) denoted by $\sigma$, i.e., $\mathcal{T}_{1}=\mathcal{T}_{\text {nom }}-\sigma$, $\mathcal{T}_{2}=\mathcal{T}_{\text {nom }}+\sigma$, such that global stability of the closedloop system (7) is ensured.

P3. Given $\mathcal{T}_{1}$ and $\mathcal{T}_{2}$, compute the maximum sector and slope bounds, such that the global stability of the closed-loop system (7) is ensured.

Note that, as $(22),(23),(24),(25)$ are LMIs, once $\mathcal{T}_{1}$, $\mathcal{T}_{2}$ and the sector bounds are fixed, these optimization problems can be straightforwardly solved by considering feasibility LMI problems and bisection techniques, where we interactively increase/decrease and test $\mathcal{T}_{2}, \sigma$ or a parameter defining the sector.

\section{NUMERICAL EXAMPLE}

Consider system (1) given by the following matrices

$$
\begin{aligned}
& A=\left[\begin{array}{cccc}
-0.5 & -6.2 & -0.105 & -1.2 \\
1 & 0 & 0 & 0 \\
0 & 1 & 0 & 0 \\
0 & 0 & 1 & 0
\end{array}\right], B_{u}=\left[\begin{array}{l}
1 \\
0 \\
0 \\
0
\end{array}\right], B_{\phi}=\left[\begin{array}{c}
0.5 \\
0 \\
0 \\
0
\end{array}\right] \\
& C=\left[\begin{array}{lll}
0 & 0.2 & 0
\end{array}\right],
\end{aligned}
$$

and the control law (4) with the following gains

$$
K_{x}=\left[\begin{array}{llll}
0.1 & 0.2 & 0.005 & 0.2
\end{array}\right], \quad K_{\phi}=[0.5],
$$

with the bounds of $\phi$ given by $\underline{\delta}=0, \bar{\delta}=\epsilon, \underline{\gamma}=-\epsilon, \bar{\gamma}=\epsilon$. In order to show the potential advantage and the conservatism reduction induced by the generic Lurie type function, we consider different structures from the function $V$ defined in (17). More specifically, we evaluate the feasibility of the inequalities such that them also holds for $V_{0}$ (i.e. $V$ with $\Lambda=0$ ), for a quadratic function of the Circle Criterion $V_{Q}(x)=x^{T} P_{11} x$, which corresponds to $V$ with $P_{12}=0, P_{22}=0, \Lambda=0$ and for the function of the Popov Criterion

$$
V_{L P}(x)=x^{T} P_{11} x+\sum_{i=1}^{m} \lambda_{i} \int_{0}^{y_{i}}\left(\phi_{i}(s)-\underline{\delta}_{i} s\right) d s,
$$

which corresponds to $V$ with $P_{12}=0, P_{22}=0$ and $\lambda_{i}>0$, $i=1, \ldots, m$. Note that for $V_{Q}$ and $V_{L P}$ we consider $P_{11} \succ 0$ and for $V_{0}$ we consider $P \succ 0$.

The obtained results for $\mathrm{P} 1$ are detailed in Table 1 , for values of $\mathcal{T}_{1}=0.1 \mathrm{~ms}$ and $\epsilon=\frac{1}{\sqrt{2}}$. For P2, we fixed $\mathcal{T}_{\text {nom }}=1.5 \mathrm{~s}$ and $\epsilon=\frac{1}{\sqrt{2}}$, which results are presented in Table 2 and for P3 we defined $\mathcal{T}_{1}=0.1 \mathrm{~ms}$ and $\mathcal{T}_{2}=2.0 \mathrm{~s}$, which results are depicted in Table 3.

Table 1. Maximum value of $\mathcal{T}_{2}$ for different Lyapunov function structures.

\begin{tabular}{|c|c|c|c|c|}
\hline & $V_{Q}$ & $V_{0}$ & $V_{L P}$ & $V$ \\
\hline $\mathcal{T}_{2}$ & 1.1072 & 1.1087 & 2.1969 & 2.6169 \\
\hline
\end{tabular}

Table 2. Maximum value of $\sigma$ for different Lyapunov function structures.

\begin{tabular}{|c|c|c|c|c|}
\hline & $V_{Q}$ & $V_{0}$ & $V_{L P}$ & $V$ \\
\hline$\sigma$ & 1.0468 & 1.0475 & 1.0861 & 1.2183 \\
\hline
\end{tabular}

Table 3. Maximum value of $\epsilon$ for different Lyapunov function structures.

\begin{tabular}{|c|c|c|c|c|}
\hline & $V_{Q}$ & $V_{0}$ & $V_{L P}$ & $V$ \\
\hline$\epsilon$ & 0.5938 & 0.5938 & 0.7798 & 1.0886 \\
\hline
\end{tabular}

In the first problem, using the generalized Lurie function $V$ we achieve values for $\mathcal{T}_{2}$ that are greater $19.1 \%, 136.0 \%$ and $136.3 \%$ than the ones obtained with $V_{L P}, V_{0}$ and $V_{Q}$, respectively. For P2, we guaranteed the stability for $\mathcal{T}_{1}=0.2817 \mathrm{~s}$ and $\mathcal{T}_{2}=2.7183 \mathrm{~s}$, with $\sigma$ greater $12.1 \%$, $16.3 \%$ and $16.3 \%$ than the ones obtained with $V_{L P}, V_{0}$ and $V_{Q}$, respectively. For the last feasibility problem, the sector bounds, represented by $\epsilon$ were $39.6 \%, 83.3 \%$ and $83.3 \%$ bigger than the ones obtained with $V_{L P}, V_{0}$ and $V_{Q}$, respectively.

To show that the achieved results support the global stabilization of the closed-loop system (7), we simulate the system considering the result from $\mathrm{P} 1$, i.e., $T_{k} \in$ [0.0001, 2.6169], and the nonlinearity

$$
\phi(y(t))=0.1 \sin (5 y(t))+0.15 y(t),
$$

that satisfies (2) with $\underline{\delta}=0, \bar{\delta}=\frac{1}{\sqrt{2}}, \underline{\gamma}=-\frac{1}{\sqrt{2}}, \bar{\gamma}=\frac{1}{\sqrt{2}}$. The control action and the states of the closed-loop system are presented in Figures 1 and 2. To obtain the values of $T_{k}$, we used a pseudo-random algorithm that generates its numbers from the standard uniform distribution on the interval previously defined.

It is easy to notice that when $t \rightarrow \infty$ the states and the control law converge to the origin, reinforcing the obtained results. 


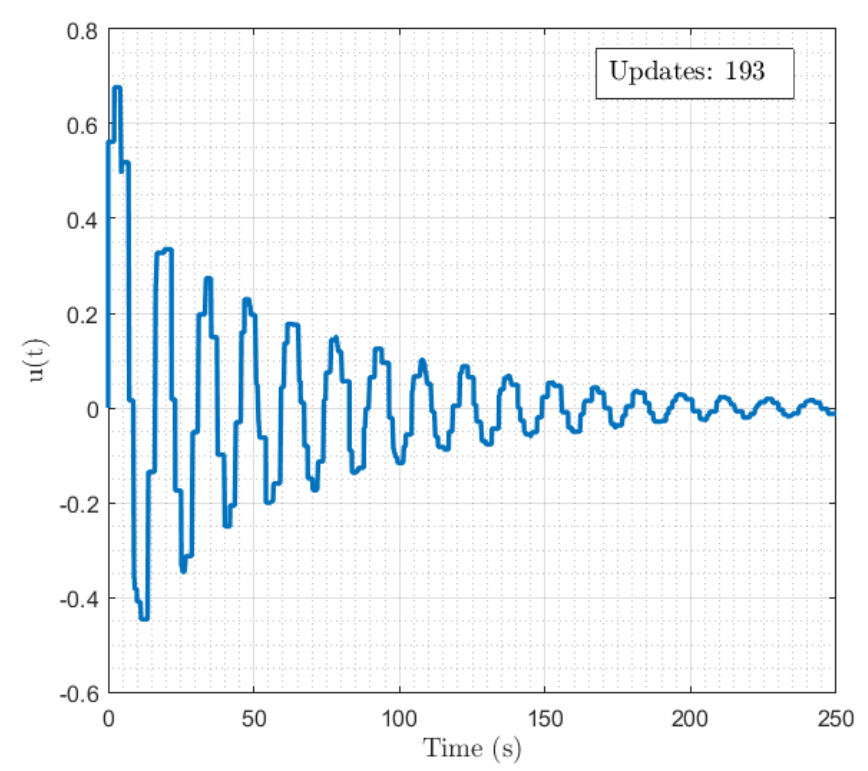

Figure 1. Control action of the closed-loop system with nonlinearity (34).

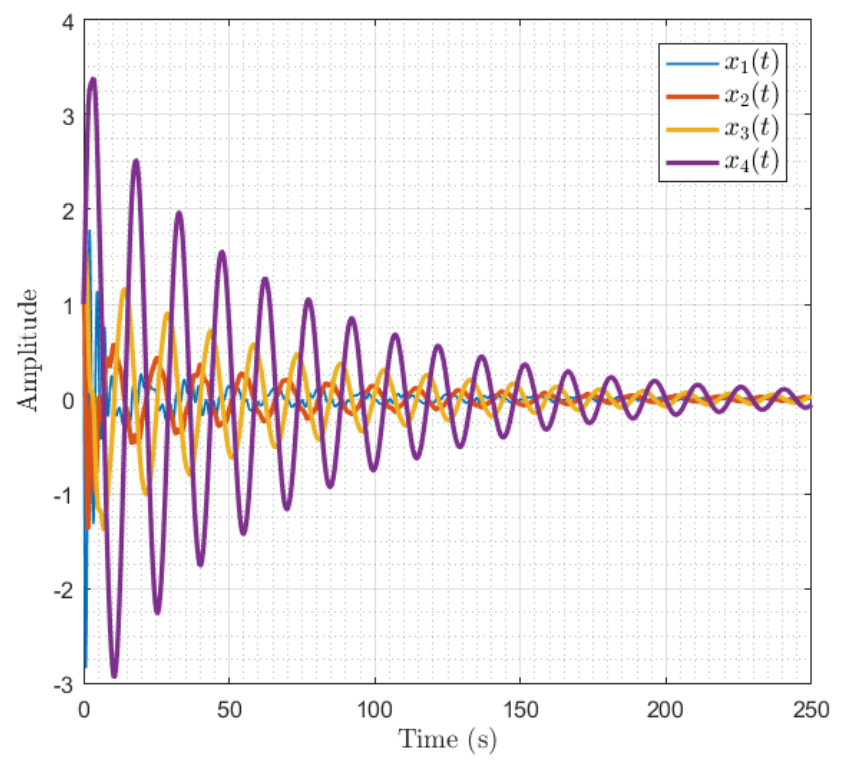

Figure 2. States of the closed-loop system with nonlinearity (34)

\section{CONCLUSIONS}

This article presents new convex conditions for global asymptotic stability of sampled-data controlled systems, subject to sector and slope-restricted nonlinearities. To develop the methods, we used the looped-functional approach and a generalized Lurie function that does not require the positivity of the matrix $P$ (as in (18)) nor the positivity of the Lurie-Postnikov coefficients.

With the presented conditions, we may solve different types of optimization problems, such as maximizing the maximum sampling time, maximizing the sampling bounds over a nominal sampling time or maximizing the sector bounds for which the GAS of the closed-loop system can be ensured.
We are currently developing local stability conditions for Lurie systems using as basis the content of this paper and later we will develop synthesis conditions for the controller. Future work also includes the study of the problem taking into account communication delays.

\section{REFERENCES}

Åström, K.J. and Wittenmark, B. (2013). Computercontrolled systems: theory and design. Dover Publications, Inc.

Bamieh, B., Pearson, J.B., Francis, B.A., and Tannenbaum, A. (1991). A lifting technique for linear periodic systems with applications to sampled-data control. Systems \& Control Letters, 17(2), 79-88.

Castelan, E.B., Tarbouriech, S., and Queinnec, I. (2008). Control design for a class of nonlinear continuous-time systems. Automatica, 44(8), 2034-2039.

Fridman, E. (2010). A refined input delay approach to sampled-data control. Automatica, 46(2), 421-427.

Fridman, E., Seuret, A., and Richard, J.P. (2004). Robust sampled-data stabilization of linear systems: an input delay approach. Automatica, 40(8), 1441-1446.

Hao, F. and Zhao, X. (2010). Absolute stability of Lurie networked control systems. International Journal of Robust and Nonlinear Control, 20(12), 1326-1337.

Hetel, L., Fiter, C., Omran, H., Seuret, A., Fridman, E., Richard, J.P., and Niculescu, S.I. (2017). Recent developments on the stability of systems with aperiodic sampling: An overview. Automatica, 76, 309-335.

Hu, L.S., Cao, Y.Y., and Shao, H.H. (2002). Constrained robust sampled-data control for nonlinear uncertain systems. International Journal of Robust and Nonlinear Control, 12(5), 447-464.

Hu, T., Huang, B., and Lin, Z. (2004). Absolute stability with a generalized sector condition. IEEE Transactions on Automatic Control, 49(4), 535-548.

Khalil, H.K. (2002). Nonlinear systems. Upper Saddle River.

Naghshtabrizi, P., Hespanha, J.P., and Teel, A.R. (2008). Exponential stability of impulsive systems with application to uncertain sampled-data systems. Systems 8 Control Letters, 57(5), 378-385.

Park, J., Lee, S.Y., and Park, P. (2018). An improved fragmentation approach to sampled-data synchronization of chaotic Lur'e systems. Nonlinear Analysis: Hybrid Systems, 29, 333-347.

Park, P. (2002). Stability criteria of sector-and sloperestricted Lur'e systems. IEEE Transactions on Automatic Control, 47(2), 308-313.

Seifullaev, R.E. and Fradkov, A.L. (2013). Sampled-data control of nonlinear oscillations based on LMIs and Fridman's method. IFAC Proceedings Volumes, 46(12), 95-100.

Seifullaev, R.E. and Fradkov, A.L. (2015). Sampled-data control of nonlinear systems based on Fridman's analysis and passification design. IFAC-PapersOnLine, 48(11), 685-690.

Seifullaev, R.E. and Fradkov, A.L. (2016). Robust nonlinear sampled-data system analysis based on Fridman's method and S-procedure. International Journal of Robust and Nonlinear Control, 26(2), 201-217.

Seuret, A. (2012). A novel stability analysis of linear systems under asynchronous samplings. Automatica, 
48(1), 177-182.

Seuret, A. and Gomes da Silva Jr., J.M. (2012). Taking into account period variations and actuator saturation in sampled-data systems. Systems \& Control Letters, 61(12), 1286-1293.

Suykens, J., Vandewalle, J., and De Moor, B. (1998). An absolute stability criterion for the Lur'e problem with sector and slope restricted nonlinearities. IEEE Transactions on Circuits and Systems I: Fundamental Theory and Applications, 45(9), 1007-1009.

Valmorbida, G., Drummond, R., and Duncan, S.R. (2018). Regional analysis of slope-restricted Lurie systems. IEEE Transactions on Automatic Control, 64(3), 12011208.

Yakubovich, V. (1965). The method of matrix inequalities in the stability theory of nonlinear control systems. ii. absolute stability in a class of nonlinearities with a condition on the derivative. Automat. Remote Control, 26, 577-592.

Zeng, H.B., He, Y., Wu, M., and Xiao, S.P. (2011). Absolute stability and stabilization for Lurie networked control systems. International Journal of Robust and Nonlinear Control, 21(14), 1667-1676.

Zhang, F., Yu, W., Wen, G., and Zemouche, A. (2019). Practical absolute stabilization of Lur'e systems via periodic event-triggered feedback. In 2019 China-Qatar International Workshop on Artificial Intelligence and Applications to Intelligent Manufacturing (AIAIM), 4247. IEEE. 